\title{
Sperm dysfunction produced by B- chromosomes in the grasshopper Myrmeleotettix maculatus
}

\author{
G. M. Hewitt, \\ T. M. East and \\ M. W. Shaw
}

School of Biological Sciences, University of East Anglia, Norwich

\begin{abstract}
The effect of B chromosomes on the process of spermatogenesis was studied in the mottled grasshopper Myrmeleotettix maculatus using light and electron microscopy. Dissections of the whole male reproductive system were stained in feulgen and scored for a variety of abnormalities, particularly in the process of organisation of sperm bundles. Many ultrastructural abnormalities were observed in the process of spermatid elongation. These problems were much more frequent in individuals with B chromosomes and caused the loss of sperm from bundles. It is suggested that B chromosomes may preferentially cause the dysfunction of spermatids carrying them and thereby produce the loss in inheritance observed in crosses. The number of sperm bundles produced decreases markedly with age.
\end{abstract}

\section{INTRODUCTION}

B chromosomes occur in a wide range of species and affect many aspects of the phenotype (see Jones and Rees, 1982 for review). They are not usually inherited in strict Mendelian ratios, and often have accumulation mechanisms which boost their frequencies when transmitted to the next generation. In well studied systems it has been shown that this tendency to increase is offset by other losses in transmission or through selection at some stage in the life cycle, and this can produce stable polymorphisms.

The mottled grasshopper Myrmeleotettix maculatus has a B-chromosome polymorphism in populations from southern Britain in which the frequency of the $B$ is increased when transmitted by the female but decreased when transmitted by the male (Hewitt 1973a, 1973b). The meiotic drive in the female is caused by the B-chromosome being distributed preferentially to the inner pole of the spindle of the 1 st meiotic division in the oocyte (Hewitt 1976). In the male the B chromosome is mitotically stable and behaves normally in meiosis. Consequently, it would seem that the loss observed in male B chromosome transmission (drag) must occur after meiosis, i.e., during spermiogenesis, sperm storage and fertilisation. The loss could not occur after this since it was possible to karyotype nearly all the eggs produced when the embryos were only a few days old. It was noticed using standard squash preparations that certain abnormal spermatid nuclei were more frequent in individuals with B-chromosomes, but the frequency was far too low to account for the loss observed (Hewitt 1973a). Several reports indicate that the process of spermatogenesis is sensitive to genetic and environmental variation. We therefore decided to examine it in detail (c.f. Roosen Runge 1977, Dumser 1980).

Spermatogenesis has been studied in some detail in Drosophila (see Lindsley and Tokuyasu 1980 for review) but is much less completely described in other insects. A general review of the male reproductive tract in insects, including Orthoptera, is given by Davey (1985), the general morphology of the reproductive system in grasshoppers is given by Uvarov (1966). There have been many chromosomal studies using Orthoptera which concentrate on just a part of spermatogenesis-usually meiosis (c.f. John and Lewis 1965, Hewitt 1979) and there have been electron microscope investigations of certain aspects of sperm ultrastructure and development (Phillips 1970, Kaye and McMaster-Kaye 1975, Szollosi $1975,1976 a, b)$.

In order to gauge the nature and extent of any effect B chromosomes might have on spermatogenesis it was first necessary to obtain a general description of the whole process in 
Myrmeleotettix maculatus. This was followed by an assessment of the differences in reproductive systems in individuals with one or more B chromosomes including an examination of the ultrastructure of abnormalities of the developing sperm.

\section{MATERIALS AND METHODS}

To examine the whole male reproductive tract, each individual was pinned through the thorax and the tip of the abdomen into a wax dish. A long cut was made along the mid-dorsal line being careful not to disturb the underlying structures. The whole body was then perfused with fresh $3: 1$ Ethanol: Acetic fixative. This procedure ensured that the testis follicles, vasa deferentia, accessory glands and sperm storage organs suffered minimum disturbance before fixation. Cuts were then made around the abdomen so that the walls could be pinned back. The reproductive tract was then dissected intact under a Nachet stereo microscope; considerable care was necessary with the vasa deferentia because they are fragile and descend into the abdominal tissues making them difficult to remove unbroken. More fixative was added as required. These tracts can be stored for months in fixative in the fridge.

The staining method we found best for differentiating the spermatids with minimum disruption to their arrangement within the tract was to hydrolyse for 5 minutes in $1 \mathrm{~N} \mathrm{HCl}$ at $60^{\circ} \mathrm{C}$ and stain with Feulgen for 30 minutes in the dark. This softened the tissues so that when the whole system was mounted on a slide in 45 per cent acetic acid it could be gently pressed under a coverslip to display the organisation of the spermatocytes, spermatids and sperm within the tract. One or two follicles can be mounted in the same way and pressed harder for more detailed analysis of their contents and for counting spermatids. The Feulgen stained the chromatin preferentially and gave very clear pictures of the nuclei. Slides were examined using low power and high power oil immersion Zeiss optics and photographs taken with a Nikon camera system.

To examine the ultrastructure of sperm development the testis follicles were combed out in saline and fixed in 4 per cent gluteraldehyde in

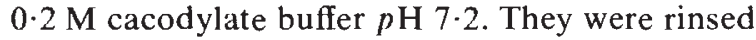
with $0 \cdot 1 \mathrm{M}$ phosphate buffer and stained with $\mathrm{OsO}_{4}$ $(0 \cdot 1 \mathrm{~g} / 10 \mathrm{ml}$ phosphate buffer $)$ for 1 hour. They were then dehydrated in an increasing concentration of acetone to 90 per cent, then stained with uranylacetate (a saturated solution in 100 per cent acetone) for 1 hour in darkness, followed by three further changes of 100 per cent acetone. Each follicle was separately embedded in firm Spurr's resin. Longitudinal or transverse sections were cut on an LKB MkII ultramicrotome using glass knives (nominal angle $55^{\circ}$ ) coated with tungsten. Thick sections $(1 \mu)$ were cut first, stained with toluidine blue and examined by light microscopy in order to locate the region of interest. Thin sections (450 $\AA$ ) were then cut for electron microscopy, collected on Formvar-coated grids and examined under a Phillips electron microscope.

Initially, individuals were used from the field and from stocks reared in the laboratory with $0 B$, $1 \mathrm{~B}$ or $2 \mathrm{~B}$ karyotypes to gain an overall picture of spermatogenesis in $M$. maculatus, and also to identify which structures, stages and parameters would be best to quantify and analyse in detail. It became clear that factors other than B karyotype such as age could be affecting the process. Consequently, the main experiment to be analysed here used males collected through the season from one population at Lakenheath Warren (Hewitt and Brown 1970), which were fixed at four different times after becoming adult. Individuals with and without Bs were included on all four occasions.

Some testes of Locusta migratoria were also fixed and examined under the electron microscope to allow comparison with the stages described by Szollosi (1975).

\section{OBSERVATIONS AND RESULTS}

The testes in $M$. maculatus are of the intermediate variety (Uvarov 1966). The left and right bundles of follicles each discharge into their own vas deferens, both of which run back to the ejaculatory apparatus from which radiates the accessory gland tubules and the left and right sperm storage organs. A composite picture of a whole system stained with Feulgen is given in fig. 1. Fig. 2 shows a single follicle from an $0 \mathrm{~B}$ individual.

At the apex of each follicle each stem cell divides asymmetrically to give a primary spermatogonium which then divides mitotically to produce cysts that normally contain $128\left(2^{7}\right)$ secondary spermatogonial cells. These growing cysts advance down the follicle and somewhat less than a quarter of the follicle is filled by these premeiotic mitoses. The cysts are frequently hexagonal in shape. They then go through the two divisions of meiosis (spermatocytes) to produce $512\left(2^{9}\right)$ spermatids per cyst.

These young spermatids then differentiate in synchrony from being round to the characteristic 


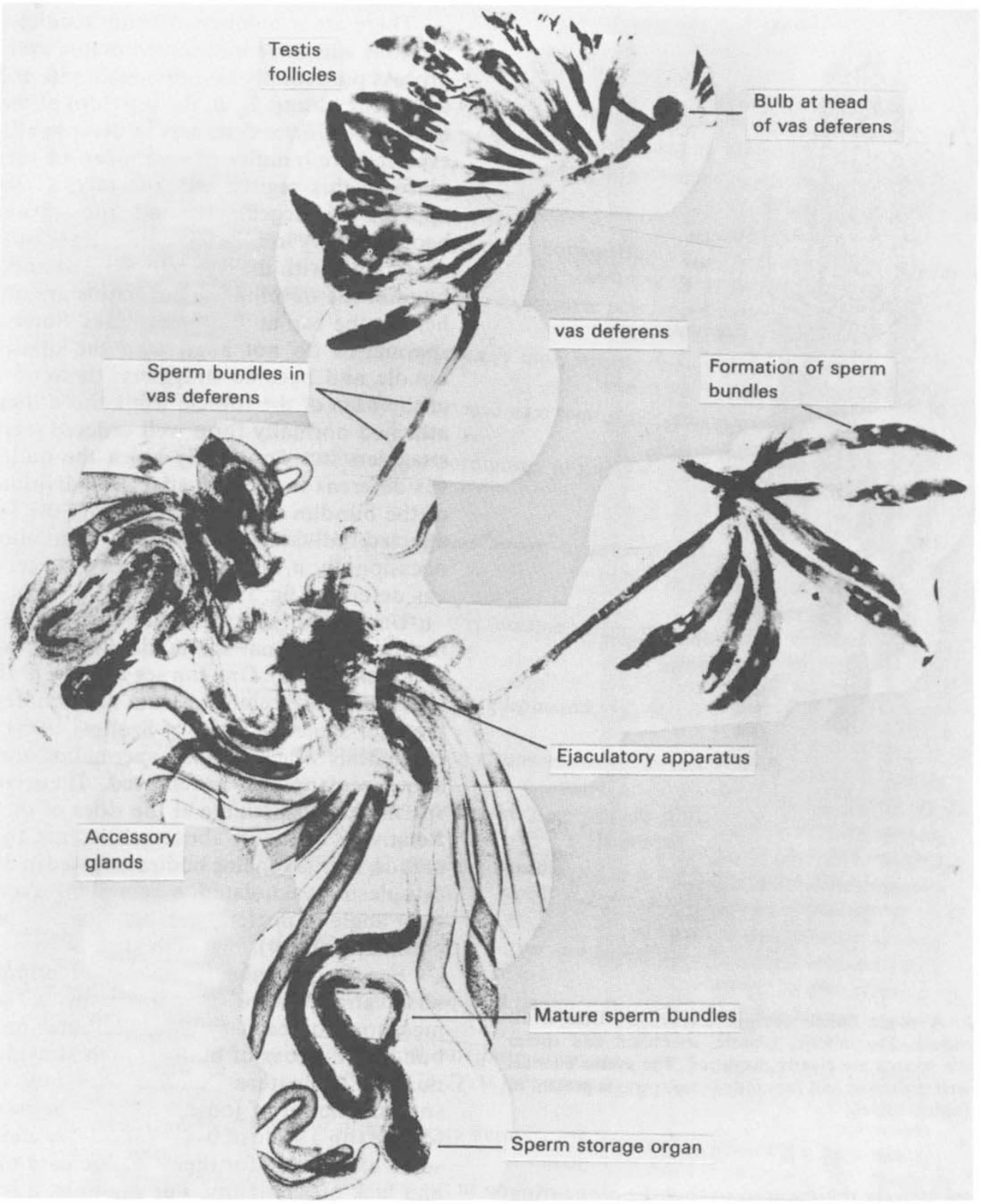

Figure 1 A composite photograph of a feulgen preparation of a bilateral reproductive tract from a male Myrmeleotettix maculatus with 2B chromosomes. Each testis comprises a bunch of follicles ( $\sim 50$ each-not all shown) which connect to the ejaculatory duct, as do the accessory gland tubules from each side (not all shown).

long nucleus at the head with a long tail. Many morphological and biochemical changes occur including the restructuring of the chromatin and proteins in the condensation of the nucleus, mitochondrial reorganisation and the production of a flagellum and axoneme. This process has been divided into 10 stages by Szollosi (1975) for Locusta migratoria and the process in M. maculatus is similar.

Each spermatid is anchored at the head by its acrosome which is embedded in the side wall of the follicle in a cap. Normally, the acrosomes of 


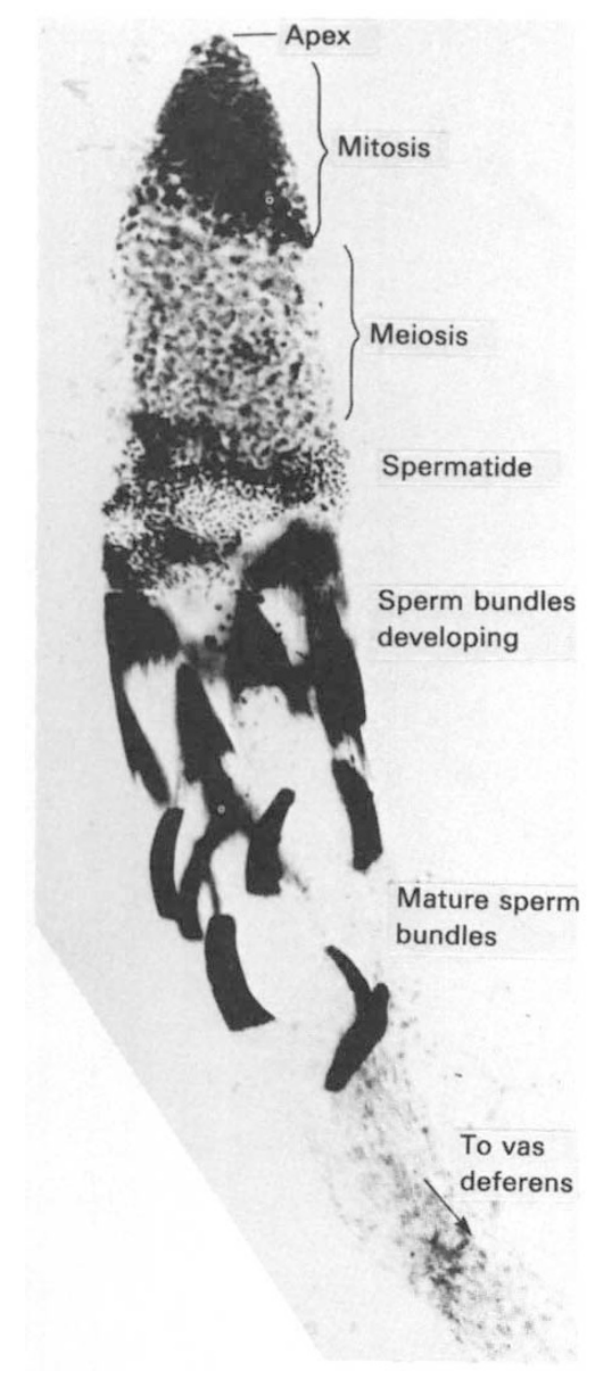

Figure 2 A single follicle feulgen preparation from a $0 \mathrm{~B}$ individual. The mitotic, meiotic, spermatid and sperm bundle sectors are clearly displayed. The sperm bundles are well organised and very little loose sperm is present in the follicle lumen.

a cyst are strictly regimented so that the elongating tails behind them produce ordered tresses of sperm. During the later stages much of the cytoplasm is sloughed off. The ordered bundle of long parallel sperm is called a spermatodesm. This undergoes a coiling. The tight bundle of sperm passes into the lumen of the follicle turning upside down so that it goes head first into the vas deferens. These bundles move away from the bulb at the blind apex of the vas deferens towards the ejaculatory duct from where they enter the seminal vesicle for storage.
There are a number of abnormalities and disruptions which were observed in this well ordered process particularly in individuals with $\mathrm{B}$ chromosomes (figs 1 and 3 ). In the top third of the follicle the progression of cysts may be disordered. Indeed, even mature bundles of sperm can be seen out of place in this region. Occasionally, a whole cyst appears to degenerate and the chromosomes become sticky in meiosis. One can see mis-shapen spermatids with the light microscope and in some bundles the developing spermatids are not tightly held in the cap at their acrosomes. Some of these spermatids do not align with the others in the bundle and become stragglers; these accumulate at the base of the follicle while those that remain attached normally form well ordered tresses. The stragglers can apparently block the outlet to the vas deferens in a badly affected individual. Some of the bundles which do pass from the follicle in affected individuals look smaller than normal and occasionally a few stragglers can be seen in the vas deferens (fig. 3).

Under the electron microscope the abnormalities in internal spermatid structure are made very clear (fig. 4). One can see how these abnormal spermatids are removed from the bundles during the later stages (stage 8 of Szollosi 1975) of spermiogenesis when the long spermatids are aligned and the cytoplasm is removed. These abnormal spermatids accumulate at the sides of the follicle. Relatively common abnormalities are twin spermatids, multiaxoneme bodies invested in discarded cytoplasm, vacuolated spermatids, axoneme at right angle to nucleus and nuclei edged with dark chromatin (fig. 4).

For the Lakenheath Warren experiment these observations were quantified in a series of measures for each follicle, viz. total number of bundles, number of bundles with stragglers, total number of mature bundles with less than 512 sperm, amount of loose sperm in the base of the follicle (on a scale of $0-4$ ). These were chosen after some experience for their relative ease of scoring and lack of ambiguity. For example, it is difficult to count with the electron microscope all the axoneme abberations in one whole follicle.

The data from the Lakenheath Warren males at four ages with $0 \mathrm{~B} 1 \mathrm{~B}$ and $2 \mathrm{~B}$ chromosomes are given in table 1 . These were analysed using the Genstat V program for regression and analysis of variance (Lawes Agricultural Trust, Rothamsted Experimental Station c. 1984). These analyses are summarised in table 2 . It is clear that individuals with B chromosomes do not have more or less bundles than those without $\mathrm{Bs}$, but that the number 

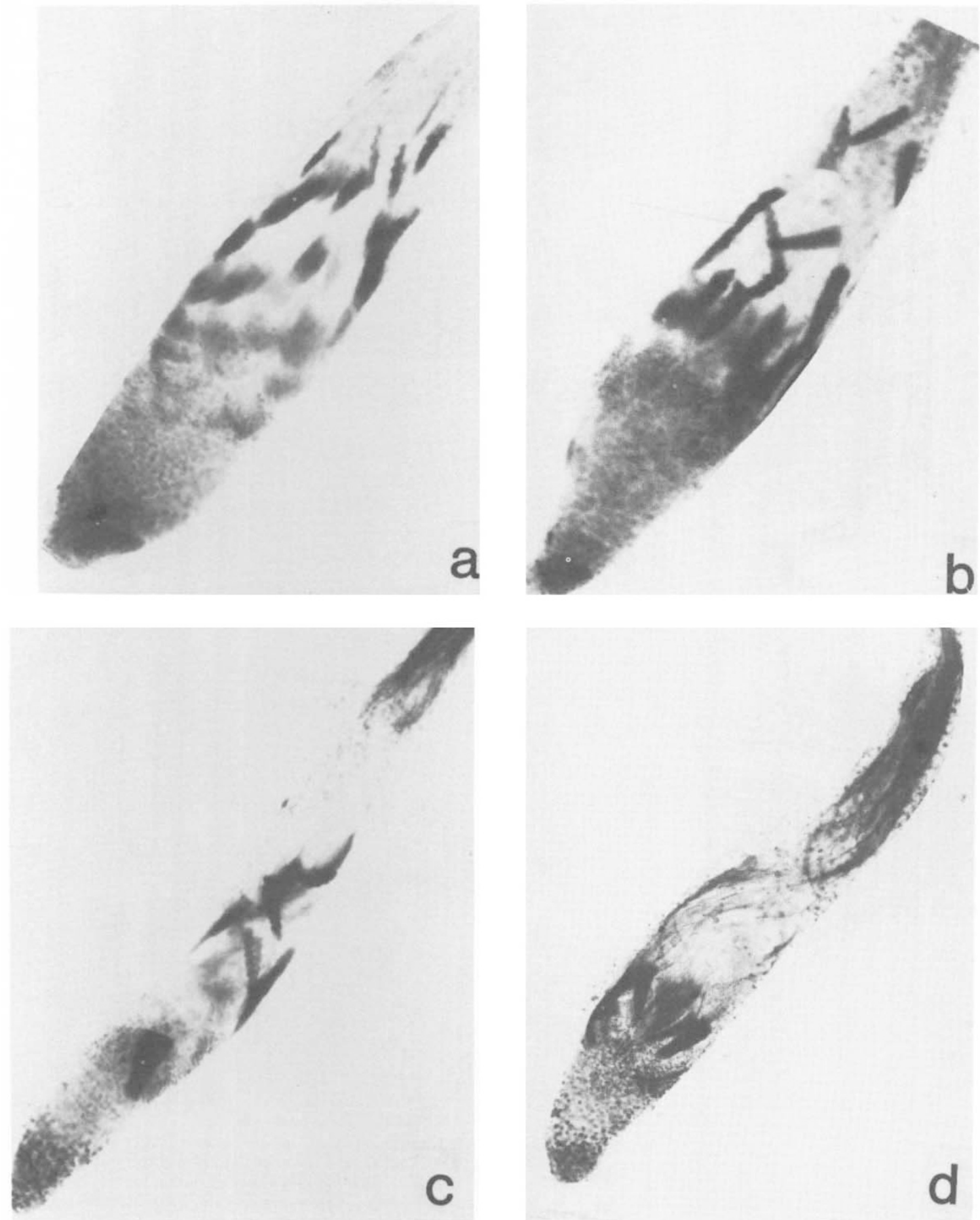

Figure 3 Four single follicle feulgen preparations a) $0 \mathrm{~B}$ individual b) $1 \mathrm{~B}$ individual c) $2 \mathrm{~B}$ individual d) $3 \mathrm{~B}$ individual. Note increasing bundle disorder and loose sperm in lumen. 

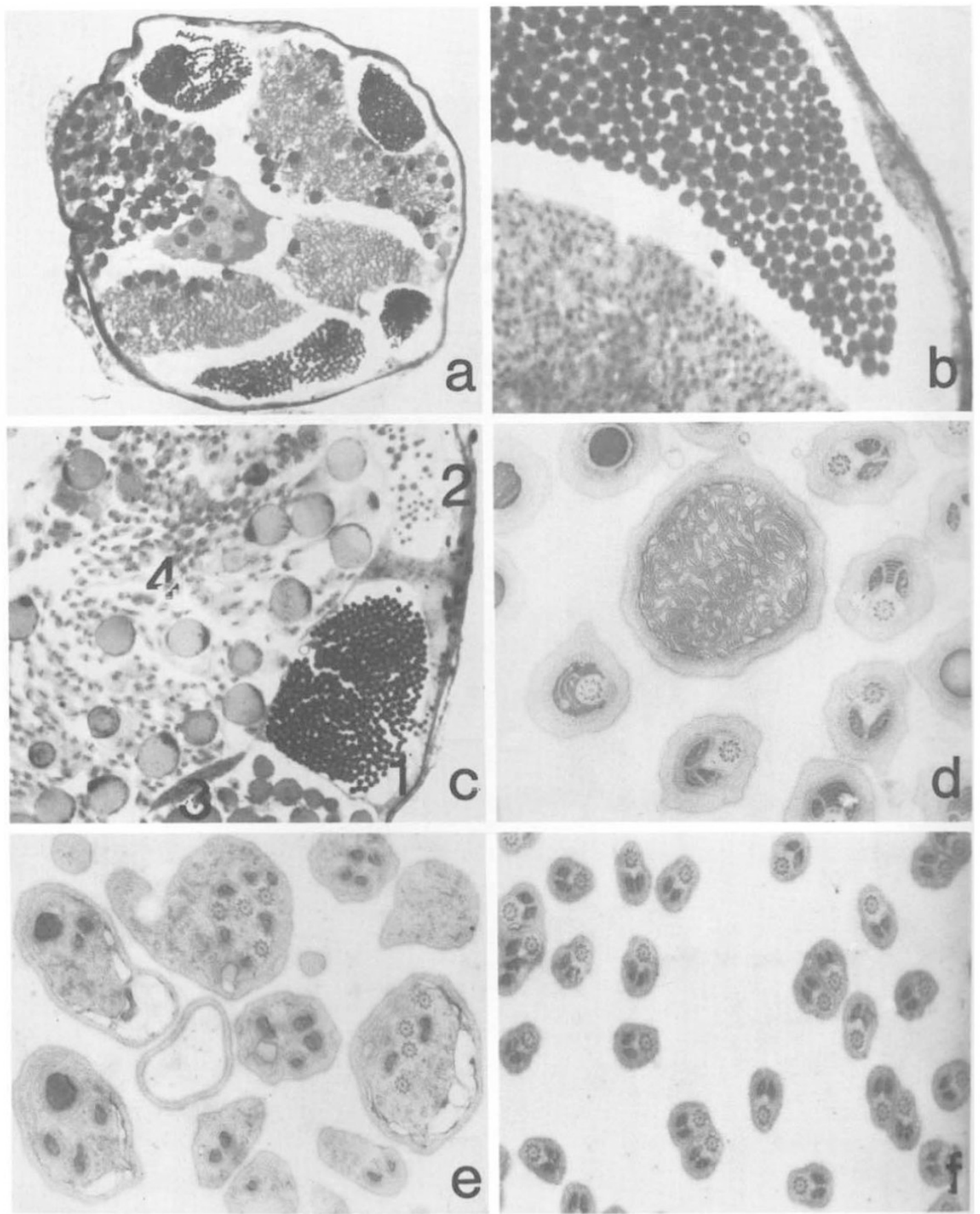

Figure 4 Abnormalities in sperm development. (a) Low power $(\times 461)$ transverse thick section $(1 \mu)$ of testis follicle from $0 \mathrm{~B}$ individual stained in toluidine blue. Dark bundles of sperm heads (stage 7-8) around the edge, lighter younger bundles showing heads and tails towards the centre. (b) Higher power $(\times 1440)$ thick section of same individual to shown regular organisation of sperm bundle of heads (stage 7) and tails (stage 5). (c) High power $(\times 1200)$ thick section from $2 \mathrm{~B}$ individual with many aberrations. (1) One dark mature bundle (stage 8) at edge of follicle containing only $350 \mathrm{sperm}$ : (2) Above this on right edge very abnormal mature bundle-few sperm of varying structure. (3) Below on bottom edge immature bundle (stage 5) showing irregular nuclei. (4) One immature bundle with heads and tails-some heads abnormal: (d) EM section $(\times 13330)$ from 2B individual showing abnormal mitochondrial derivatives and one large head nucleus. (e) EM section $(\times 9860)$ of $1 \mathrm{~B}$ individual showing several abnormal spermatids (stage 6) with multiple nuclei and axonemes. (f) EM section $(\times 9730)$ of $1 \mathrm{~B}$ individual showing a mixture of normal and abnormal spermatids (stage 8 ). 


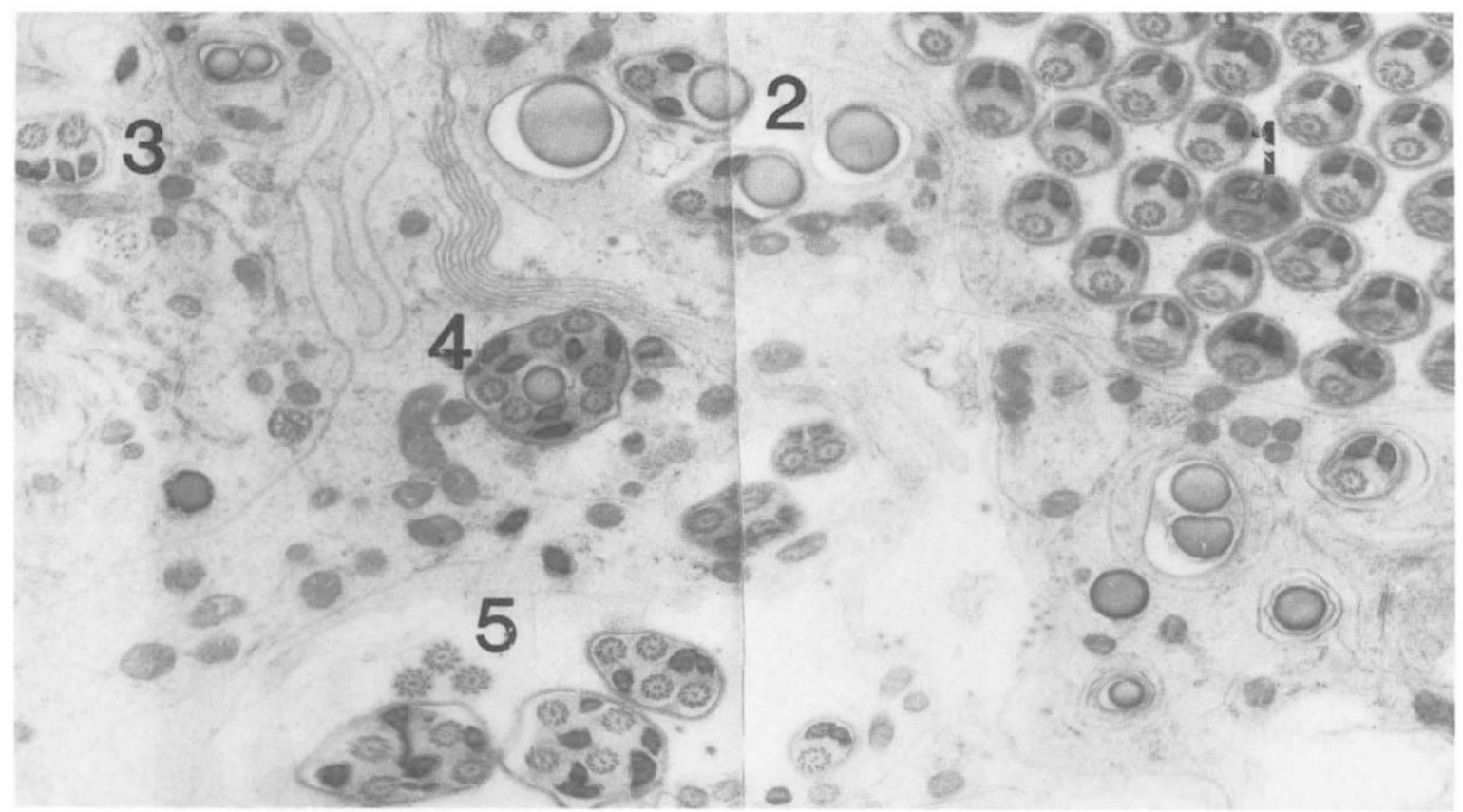

Figure 5 EM section of 1B individual ( $\times 13720$ ) showing (1) normal spermatid bundle (stage 9) (2) abnormal overlap of nucleus and tail (3) twin spermatid (4) conglomerate of 6 axonemes and 6 mitochondrial derivatives with one nucleus (5) three loose axonemes. The left side of the picture shows the sloughing away of cytoplasm and abnormalities that occurs between stages $4-8$.

of bundles per follicle decreases markedly as the insects age (table $2 \mathrm{a}$ ). Individuals with $\mathrm{B}$ chromosomes have more loose sperm in the follicle lumen than individuals without Bs (table $2 \mathrm{~b}$ ); there is an increase with age (Date) that is significant at the 5 per cent level, and there is also an interaction between B chromosomes and Date which is just significant at 5 per cent.

The analysis of the third and fourth metricsnumber of small bundles (SB) and number of bundles with stragglers (BS) are slightly different, because SB and BS are determined by the number of bundles (NB) which is itself significantly affected by Date (table 2a). Consequently the effect of the covariate NB is removed first in the analysis of SB or BS (tables $2 \mathrm{c}$ and $2 \mathrm{~d}$ line 1). The analysis of the remaining variance shows that the number of small bundles (SB) is greatly increased by $B$ chromosomes and there is a significant interaction of B chromosomes and Date. This interaction is largely due to the figures from $2 \mathrm{~B}$ individuals which in earlier dates were proportionately larger than $\mathrm{OB}$ and $1 \mathrm{~B}$ but on later dates were smaller. This may in part be due to the paucity of data on later dates, which itself is due to a low frequency and low survival of insects of the 2B karyotype. The analysis of the remaining variance for number of bundles with stragglers (BS) gives a strong indica- tion that B chromosomes increase their number but this is not quite formally significant at 5 per cent level, and neither is its interaction with Date. No significant difference was found between $0 \mathrm{~B}$, $1 \mathrm{~B}$ and $2 \mathrm{~B}$ testes in the number of follicles comprising them $(\bar{x}: 0 B=51 \cdot 0 \pm 6 \cdot 552,1 B=49 \cdot 0 \pm 5 \cdot 69$, $2 B=47 \cdot 66 \pm 3 \cdot 78)$ although there is a slight downward trend (cf. Lopez-Fernandez and Gosalvez 1982).

\section{DISCUSSION}

These results show that $\mathrm{B}$ chromosomes have major effects on spermiogenesis: they cause an obvious dysfunction that can be measured by more sperm bundles with reduced numbers of sperm, probably more bundles with disarranged and straggling sperm, and more loose sperm in the lumen of the follicle. Furthermore many structural aberrations become apparent under the electron microscope, particularly when the cytoplasm is being removed from the maturing sperm. These losses from the bundles accumulate in the lumen of the follicle, but few if any aberrant sperm are transported to the storage organ along with the regularly coiled bundles. It seems likely then that the B chromosome causes a reduction in the total number of sperm produced. 
Table 1 Data from the Lakenheath Warren males sampled at 4 ages (Date) and containing 0B, 1B and 2B individuals. Each individual was averaged over 10-20 follicles to give measures per follicle for (a) number of bundles (NB) (b) loose sperm on a scale 0-4 (LS) (c) number of small bundles, <512 spermatids (SB) (d) number of bundles with stragglers (BS)

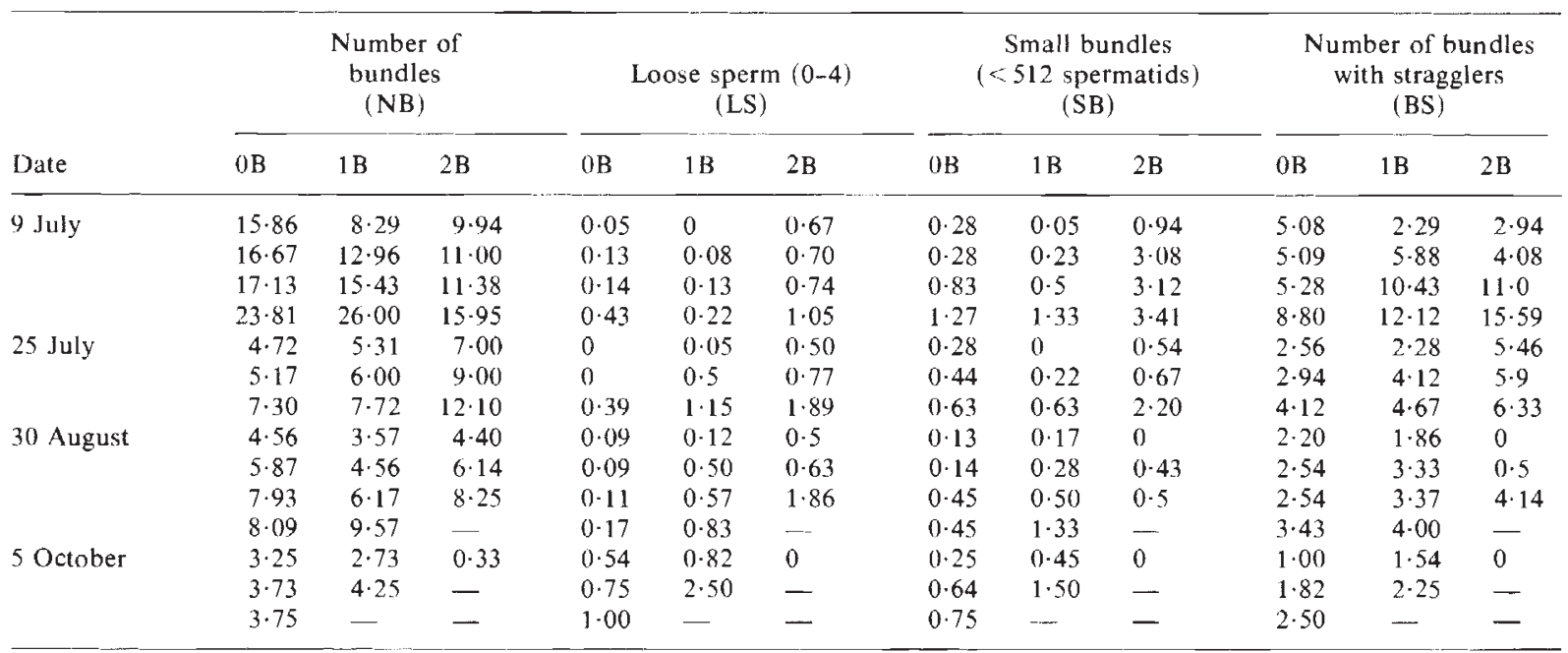

Table 2 Analysis of variance of data in Table 1 for $0 \mathrm{~B}, 1 \mathrm{~B}$ and $2 \mathrm{~B}$ individuals ( $\mathrm{B}$ chrom) on dates 1 to 4 (Date). (a) Number of bundles per follicle (NB), (b) Loose sperm (LS), (c) Small Bundles $<512$ sperm (SB), (d) Number of bundles with Stragglers (BS). The $c$ and $\mathrm{d}$ analyses include the covariate for numbers of bundles (NB)

\begin{tabular}{|c|c|c|c|c|c|}
\hline \multicolumn{6}{|l|}{ a) $\mathrm{NB}$} \\
\hline Item & $\mathrm{dF}$ & $\mathrm{SS}$ & MS & VR & $P$ \\
\hline B Chrom & 2 & 1.90 & 0.95 & 0.09 & NS \\
\hline Date & 3 & $828 \cdot 08$ & 276.03 & $24 \cdot 89$ & $<0.001^{* * *}$ \\
\hline B chrom $\times$ Date & 6 & $95 \cdot 44$ & $15 \cdot 91$ & $1 \cdot 43$ & $>0 \cdot 2$ \\
\hline $\begin{array}{l}\text { Residual } \\
\text { b) LS }\end{array}$ & 26 & $288 \cdot 38$ & 11.09 & & \\
\hline ltem & $\mathrm{dF}$ & SS & MS & VR & $\mathrm{P}$ \\
\hline B chrom & 2 & $2 \cdot 0095$ & 1.0047 & $5 \cdot 32$ & $0.01^{*}$ \\
\hline Date & 3 & $1 \cdot 8470$ & 0.6157 & $3 \cdot 26$ & $0.05-0.01^{*}$ \\
\hline$B$ chrom $\times$ Date & 6 & $3 \cdot 2382$ & 0.5397 & $2 \cdot 86$ & $0.05^{*}$ \\
\hline $\begin{array}{l}\text { Residual } \\
\text { c) SB }\end{array}$ & 26 & $4 \cdot 9070$ & $0 \cdot 1887$ & & \\
\hline Item & $\mathrm{dF}$ & SS & MS & VR & $P$ \\
\hline NB & 1 & $4 \cdot 5641$ & $4 \cdot 5641$ & $23 \cdot 48$ & $<0.001^{* * *}$ \\
\hline B chrom & 2 & 5.6892 & $2 \cdot 8446$ & $14 \cdot 63$ & $<0.001^{* * * *}$ \\
\hline Date & 3 & 1.0938 & 0.3646 & $1 \cdot 88$ & $>0 \cdot 2$ \\
\hline B chrom $\times$ Date & 6 & $11 \cdot 2642$ & 1.8774 & $9 \cdot 66$ & $<0.001^{* * *}$ \\
\hline $\begin{array}{l}\text { Residual } \\
\text { d) } \mathrm{BS}\end{array}$ & 25 & $4 \cdot 8594$ & $0 \cdot 1944$ & & \\
\hline Item & $\mathrm{dF}$ & SS & MS & VR & $P$ \\
\hline NB & 1 & $254 \cdot 843$ & $254 \cdot 843$ & 71.96 & $<<0.001^{* * * *}$ \\
\hline B chrom & 2 & $19 \cdot 408$ & $9 \cdot 704$ & $2 \cdot 74$ & $0 \cdot 1-0.05$ \\
\hline Date & 3 & 7.951 & $2 \cdot 650$ & 0.75 & NS \\
\hline B chrom $\times$ Date & 6 & $50 \cdot 300$ & $8 \cdot 383$ & $2 \cdot 37$ & $0 \cdot 1-0 \cdot 05$ \\
\hline Residual & 25 & $88 \cdot 542$ & $3 \cdot 542$ & & \\
\hline
\end{tabular}

On the other hand, the number of bundles produced is not altered by the presence of $\mathrm{B}$ chromosomes, so the factors governing the rates of apical and premeiotic mitoses are probably not affected ( $c f$ Lindsley and Tokuyasu 1980). As the grasshopper gets older fewer bundles are produced, that is there is no "steady state" and the rate of production is declining throughout adult life. Increasing age also brings more loose sperm in the follicle, but not a higher proportion of small bundles and bundles with stragglers. 
The significant interaction terms between $\mathrm{B}$ chromosomes and Date are produced by differences between the age samples in the effects of the karyotypes. There is considerable variation between individuals and the process of spermiogenesis would seem to be affected by other factors both genetic and environmental, e.g., Szollosi $(1976 a, b)$ has shown how temperature affects spermiogenesis in locusts. One possibility to explain some of the interaction effect could be that individuals with $0 \mathrm{~B}, 1 \mathrm{~B}$ or $2 \mathrm{~B}$ karyotypes mature and age at different rates. Thus whilst all individuals are of similar chronological age $B$ individuals may be "younger" at the beginning of the experiment but "age" more quickly than $0 \mathrm{~B}$ individuals in terms of their spermiogenesis. We have some evidence for different rates of development in embryonic and nymphal stages, and for different adult survivals between B karoytypes (Hewitt and East 1978, Harvey and Hewitt 1979, Hewitt $1973 b$ ).

It is pertinent to ask just how the $\mathrm{B}$ chromosome is producing this array of effects which appears to be an increase in the abnormalities seen to a lesser extent in $\mathrm{OB}$ individuals. These include abnormalities of nuclear and microorganellar ultrastructure, spermatids not attaching by their heads and coming loose from the bundles and problems with bundle transport. The abnormalities seen under the electron microscope are particularly apparent when the cytoplasm is being sloughed away in the later stages of spermiogenesis. But the signal for this may be well in advance of this time and produce the visible result by a sequence of events (Lindsley and Tokuyasu 1982). Now B chromosomes are known to modify meiotic processes such as recombination (John and Hewitt 1965, Jones and Rees 1982) and this may affect later stages of spermiogenesis. Peacock and Miklos (1973) suggested a "pairing-abnormal" spermatid hypothesis from information on Drosophila. Miklos (1974) extended this to include the B chromosome system of $M$. maculatus since the level of non-disjunction (caused by no chiasmata) was correlated with the loss of B's in the progeny. The disruption we have observed in spermiogenesis may be the linking mechanism.

On the other hand, B's are known to affect an individual's development in a number of ways so that the animal's whole testicular physiology may be somewhat unbalanced. It is possible that such a general effect may produce the abnormalities rather than a specific effect at meiosis.

Of particular importance is the possibility that the $\mathrm{B}$ chromosomes are producing the abnor- malities preferentially in those spermatids carrying them rather than in their 0B fellows. This could! be achieved in a number of ways. The extra mass of DNA added by the B chromosome seems an unlikely cause since in a normal male with an $\mathrm{XO}$ sex mechanism half the spermatids have a large $X$. chromosome and the other half do not. It could be that the B chromosome DNA is transcribed and active in the spermatid producing molecules that are detrimental either by their nature or quantity. However, in general B's seem fairly inert in the systems and tissues where they have been examined, and there is little synthetic activity in spermatids (Jones and Rees 1982, Fox et al. 1979). Perhaps a more likeíy possibility is that the B chromosome interferes in the extensive molecular and structural rearrangements that occur in the nucleus of the developing spermatid (Kaye and McMaster-Kaye 1975). In particular, it has been shown in the segregation distorter (SD) system in Drosophila melanogaster using a fluorescent dye that the dysfunctional sperm are those which do not make the normal change from somatic to spermatid-specific histones in spermiogenesis (Hauschteck-Jungen and Hartl 1982). Interestingly, it has been demonstrated that B chromosomes have a different DNA-protein relationship from autosomes in some plants (Jones and Rees 1982) and this may cause problems in histone: transition during spermiogenesis.

Clearly, the presence of B chromosomes is causing sperm dysgenesis which may well account: for the loss of B's in transmission through the male side in crosses (Hewitt 1973a). To prove conclusively that the abnormal sperm are those that contained B chromosomes would require a specific: probe for B chromosome DNA and as yet this is not available. However, we do have some correlative evidence which supports this hypothesis. In another series of experiments (Hewitt and East, unpublished) involving some 50 single pair crosses: to determine the transmission rates of B chromosomes, follicles were taken for EM analysis from the surviving males. For three of these, which were $1 \mathrm{~B}$ males mated with $\mathrm{OB}$ females, we have data on the average number of spermatids per bundle from EM sections and B-transmission rate, and there is a correlation between the two measures. (Transmission: Spermatids per bundle, 0.70:399, $0.48: 294,0.05: 220$ ). These data are sparse-they are difficult to acquire-but they suggest that $\mathrm{B}$ chromosomes are lost by causing the preferential dysfunction of the spermatids that carry them.

Even though the presence of B chromosomes reduces the number of sperm produced, particularly 
in $2 \mathrm{~B}$ and $3 \mathrm{~B}$ individuals, it is difficult to gauge the effect of this on fertility. Each sperm bundle contains more than enough sperm to fertilise all the eggs a female lays in her lifetime, and a spermatophore transfers many bundles at a single mating. Therefore B chromosomes will not be important to male fertility for a single mating. However, it is becoming clear that female grasshoppers can mate several times (Butlin, Hewitt, Mason and Ritchie unpublished) and consequently the nature and extent of sperm competition will decide what loss of male fecundity the B chromosomes cause. It could be very significant.

Acknowledgements We are grateful to the S.E.R.C. and N.E.R.C. for financial support and to Dr Roger Butlin for advice on the manuscript.

\section{REFERENCES}

DAVEY, K. G. 1985. The male reproductive tract. In Comparative Insect Physiology, Biochemistry and Pharmacology Vol. 1. Kerkut, G. A. and Gilbert, L. I. (eds.), Pergamon Press, Oxford, pp. 1-14.

DUMSER, J. B. 1980. The regulation of spermatogenesis in insects. Ann. Rev. Entomol., 25, 341-369.

FOX, D. P., HEWITT, G. M. AND HALI, D. J. 1974. DNA replication and RNA transcription of euchromatic and heterochromatic chromosome regions during grasshopper meiosis. Chromosoma, 45, 43-62.

HAUSCHTECK-JUNGEN, E. AND HARTL, D. L. 1982. Defective histone transition during spermiogenesis in heterozygous segregation distorter males of Drosophila melanogaster. Genetics, 101, 57-69.

HARVIY, A. W. AND HEWITT, G. M. 1979. B-chromosomes slow development in a grasshopper. Heredity, 42, 397-401.

HEWITT, G. M. 1973a. Variable transmission rates of a Bchromosome in Myrmeleotettix maculatus (Thunb.) (Acrididae: Orthoptera). Chromosoma, 40, 83-106.

HEWITT, G. M. $1973 \mathrm{~h}$. The integration of supernumerary chromosomes in the Orthopteran genome. Cold Spring Harb. Symp. quant. Biol., 38, 183-194.

HEWITT, G. M. 1976. Meiotic drive for B-chromosomes in the primary oocytes of Myrmeleotettix maculatus (Orthoptera: Acrididae). Heredity, 35, 375-387.
HEWITT, G. M. 1979. Orthoptera-grasshoppers and crickets Animal Cytogenetics, 3, Insecta Part 1. Borntraeger. Stuttgart.

HIWITT, G. M. AND BROWN, F. M. 1970. The B-chromosome system of Myrmeleotettix maculatus V. A steep cline in East Anglia. Heredity, 25, 363-371.

HEWIIT, G. M. AND EAS I, I. M. 1978. Effects of B chromosomes on development in grasshopper embryos. Heredity, 41, 347-356.

JOHN, B. AND HEWITT, G. M. 1965. The B-chromosome system of Myrmeleotettix maculatus (Thunb.) I. The Mechanics. Chromosoma (Berl.), 16, 548-578.

JOHN, B. AND LEWIS, K. R. 1965. The Meiotic System. Protoplasmatologia VI. F. 1. Springer-Verlag. Wien.

JONES, R. N. AND REES, H. 1982. B chromosomes. Academic Press, London, p. 226.

KAYI, J. S. AND McMASTER-KAYE, R. 1975. The fine structure and protein composition of developing spermatid nuclei. In The Biology of the Male Gamete. Duckett, J. G. and Racey, P. A. (eds.) Biol. J. Linn. Soc. 7, Suppl. 1, Academic Press, pp. 227-237.

LINDSLEY, D. L. AND TOKUYASU, K. T. 1980. Spermatogenesis. In The Genetics and Biology of Drosophila. Vol. $2 d$. Ashburner, M. and Wright, T. R. F. (eds.), Academic Press, London, Ch. 32. pp. 226-294.

LOPEZ-FERNANDEZ, C. AND GOSALVEZ, J. 1982. Detection of abnormal spermatid nuclei in Orthoptera by silver staining. Cytobios, 34, 71-74.

MIKLOS, G. L. G. 1974. Sex-chromosome pairing and male fertility. Cytogenet. Cell Genet., 13, 558-577.

PI:ACOCK, W. J. AND MIKLOS, G. L. G. 1973. Meiotic drive in Drosophila: new interpretations of the segregation distorter and sex chromosome systems. Advances in Genetics, 17, 361-409.

PHILliPS, D. M. 1970. Insect Sperm: their structure and morphogenesis. J. Cell Biol., 44, 243-277.

ROOSEN-RUNGE, E. C. 1977. The Process of Spermatogenesis in Animals. Cambridge University Press. Cambridge.

SZOLLOSI, A. 1975. Flectron microscope study of spermiogenesis in Locusta migratoria (Insecta: Orthoptera). $J$ Ultrastruct. Res., 50, 322-346

SZOLLOS1, A. $1976 a$. Influence of infra-optimal breeding temperature on spermiogenesis of the locust Locusta migratoria 1. Abnormalities in differentiation of the cytoplasmic organelles. J. Ultrastruct. Res., 54, 202-214.

SZOLLOSI, A. $1976 \mathrm{~b}$. Influence of infra-optimal breeding temperature on spermiogenesis of the locust Locusta migratoria II. Abnormalities in differentiation of the nucleus. J. Ultrastruct. Res., 54, 215-223.

UVAROV, B. 1966. Grasshoppers and Locusts. Vol. I. Cambridge University Press. Cambridge. 